\title{
New Stochastic Network Calculus for Loss Analysis
}

\author{
A. Gulyás, J. Bíró, and F. Németh \\ Budapest University of Technology and Economics \\ Email: \{gulyas,biro,nemethf\}@tmit.bme.hu
}

\begin{abstract}
Recently some stochastic (probabilistic) extensions of the deterministic network calculus have been developed, mainly for exploiting the statistical multiplexing of flows aggregated in packet based communication networks. This exploitation could result "better" stochastic performance bounds than those bounds provided by the inherently worst case analysis of the deterministic network calculus. The core of these stochastic extensions is the re-definition of the so-called arrival and service curve in a probabilistic manner. Until this time the re-definitions of these curves are based on tail probability like functionals. In this paper we perform a new kind of stochastic network calculus based on defining arrival and service curves using a different functional called tail weight. The power of this approach is demonstrated by presenting fundamental results on backlog and delay bounds and concatenation of nodes, furthermore suitable service curves and numerical examples are also presented for one of the most complicated packet service disciplines, the generalized processor sharing scheduler.
\end{abstract}

\section{Keywords}

stochastic network calculus, workload loss ratio, statistical multiplexing

\section{INTRODUCTION}

In this paper we perform a new kind of stochastic network calculus based on defining arrival and service curves using a different functional called tail weight. The application of this functional is two-fold. On one hand, a very similar

Permission to make digital or hard copies of all or part of this work for personal or classroom use is granted without fee provided that copies are not made or distributed for profit or commercial advantage and that copies bear this notice and the full citation on the first page. To copy otherwise, to republish, to post on servers or to redistribute to lists, requires prior specific permission and/or a fee.

VALUETOOLS 2009, October 20-22, Pisa, Italy

Copyright () 2009 ICST 978-963-9799-70-7

DOI 10.4108/ICST.VALUETOOLS2009.7752 functional can be used to estimate the loss rate of traffic, hence, this approach might inherently be suitable for loss performance analysis in communication networks. On the other hand, this functional is fully conformant to the useful stochastic ordering of random variables by convex functions. This type of stochastic ordering turned out to be extremely efficient for calculating the new type of arrival and service curves. The power of this approach is demonstrated by presenting fundamental results on backlog and delay bounds and concatenation of nodes, furthermore suitable service curve is also presented for one of the most complicated packet service disciplines, the generalized processor sharing scheduler.

The results of this paper are performed (without restrictions, only for presentation purposes) in the discrete time domain (having $t=1,2, \ldots$ discrete time instants) and the following notations are used: $A(s, t]$ denotes the number of bits arrived to a node from a flow and $D(s, t]$ is the output flow from the node within the interval $(s, t]$. If $A(t)$ and $D(t)$ are used that will mean $A(0, t]$ and $D(0, t]$, respectively. The backlog at time $t$ is given by $B(t)=A(t)-D(t)$ and the delay at time $t$ is given by $W(t)=\inf \{d \geq 0: A(t-d) \leq D(t)\}$. In a network context let $A^{N}(t)$ and $D^{N}(t)$ denote the arrivals and departures at node $N$. Finally, let the positive part operator be defined as $(e x p r)^{+}=\max [\operatorname{expr}, 0]$.

In this paper $A$ and $D$ are assumed to be stationary and ergodic processes, no further assumptions are imposed.

\section{DETERMINISTIC NETWORK CALCU- LUS}

The original deterministic network calculus [25] operates with envelope functions defined in a deterministic manner to describe arrivals and services. The typical calculations are built around a set of well-constructed algebraic manipulations, which are coined as min-plus and max-plus algebra [3].

In data communication networks two operations on the minplus algebra has a particular importance. In what follows the original definitions from [16] are recalled.

Definition 1 (Min-Plus Convolution). Let $f$ and $g$ be two wide-sense increasing ${ }^{1}$ functions. The min-plus con-

${ }^{1}$ A function $f$ is wide-sense increasing if and only if $f(s) \leq$ $f(t)$ for all $s \leq t$. 
volution of $f$ and $g$ is the function $f \otimes g(t)=\inf _{0 \leq s \leq t}\{f(t-$ $s)+g(s)\}$.

Definition 2 (Min-Plus DeCONVOlution). Let $f$ and $g$ be two wide-sense increasing functions. The min-plus deconvolution of $f$ and $g$ is the function

$$
f \oslash g(t)=\sup _{0 \leq u}\{f(t+u)-g(u)\} .
$$

The deterministic envelope function for the arrival process is called arrival curve and its definition is as follows [16]:

Definition 3 (Arrival CuRve [16]). An arrival process $A(t)$ has an arrival curve $\alpha$, if

$$
A(t)-A(s) \leq \alpha(t-s) \Leftrightarrow A \leq A \otimes \alpha .
$$

Definition 4 (Service CuRve [16]). Consider a node $N$ and a flow through $N$ with input and output functions $A(t)$ and $D(t)$ respectively. We say that $N$ offers to the flow a service curve $\beta$ if and only if

$$
D(t) \geq A \otimes \beta(t) .
$$

The power of deterministic network calculus lies in the usage of the end-to-end (network) service curve, which can provide much better results in performance bounds, than that of analyzing the nodes in isolation and simply sum up the per node bounds. If the $h$ th node within the route $(h=1,2, \ldots, H)$ of nodes offers to a flow a service curve $\beta_{h}$, then the network service curve can be expressed as $\beta_{\text {net }}=$ $\beta_{1} \otimes \beta_{2} \otimes \ldots \otimes \beta_{H}$.

\section{STOCHASTIC EXTENSIONS OF THE DE- TERMINISTIC NETWORK CALCULUS}

Although the deterministic network calculus is a powerful and expressive tool for describing the properties of communication networks, its worst-case system view cannot take the effects of the statistical multiplexing into consideration. This fact usually leads to the overestimation of the resource requirements of multiplexed traffic sources. In order to utilize the statistical multiplexing phenomenon, the extension of network calculus in a stochastic manner is a valuable research directions in performance evaluation tools and methodologies.

\subsection{Saturation (tail) Probability Versus Loss Ratio}

Recently, several proposals have been made $[21,2,4,22,27$, $9]$ to overcome the weaknesses of the original deterministic network calculus, most of them concentrating on adding stochastic functionality to the original deterministic rules (inequalities (2) and (3)). The so called effective envelope approach achieves it in the following way.

$$
P\left\{A(t+\tau)-A(t)>E^{\varepsilon}(\tau)+x\right\} \leq \varepsilon(x)
$$

and for services:

$$
P\left\{A \otimes S^{\varepsilon}(t)>D(t)+x\right\} \leq \varepsilon(x)
$$

In (4) and (5) the terms $E^{\varepsilon}$ and $S^{\varepsilon}$ are called as arrival and service envelopes with violation probability $\varepsilon$. The term $P\left\{A(t+\tau)-A(t)>E^{\varepsilon}(\tau)+x\right\}$ is called tail or saturation probability. This approach is useful since a set of QoS requirements can be expressed in the form of tail probabilities which provides a convenient description of the investigated systems, at least in shorter time scales. For example the buffer saturation probability in queueing models of network nodes is often proposed as a Quality of Service measure [7, $15,11,25,24,23$. In numerous queueing theoretical studies the buffer saturation probability (in infinite buffer systems) is analyzed $[26,1,18,6,5,11,25,24,23]$ and its estimates are promoted as built-in elements in Quality of Service architectures. This measure quantifies the fraction of time during which the buffer occupancy exceeds a certain threshold, and bounds from above the fraction of time during the finite buffer (with the size of the threshold) is full. ${ }^{2}$

Although with the effective envelope approach the saturation type network characteristics can be easily derived, it faces some problems when important long-time behavior characteristics such as workload loss ratio are to be quantified. The buffer overflow probability is frequently used for loss ratio estimation $[7,15,25,24,23]$, nevertheless, it is shown in [11], that the ratio of the workload loss ratio (WLR) and the buffer saturation probability can be arbitrary under certain circumstances. The expected traffic (packet) loss ratio at network nodes or a network of nodes is one of the key QoS parameters which should always be considered and controlled in almost all kind of traffic. Traffic management functions (like connection admission control, packet scheduling algorithms) and network designing methods strongly rely on loss performance analysis.

The characteristics of a system in longer time scales can be conveniently expressed with a different stochastic extension of the inequalities (2) and (3). This approach has been previously examined in [10], however, the statements presented there are not valid for the entire sample path. The main result of this paper is the introduction of this novel type of probabilistic extension completed with a time adaptive approach that is used in sample path arguments.

The novel definitions will take the following form with the socalled tail weight functional : $E\left[\left(A(t+\tau)-A(t)-Z^{\varphi}(\tau)\right)^{+}\right] \leq$ $\varphi$. for arrivals and $E\left[\left(A \otimes S^{\varphi_{s}}(t)-D(t)\right)^{+}\right] \leq \varphi_{s}$. for services. Between the left hand side of (4) and the tail weight functional above the following relation holds $E[(A(t+\tau)-$ $\left.\left.A(t)-Z^{\varphi}(\tau)\right)^{+}\right]$

$=\int_{x=0}^{\infty} P\left(A(t+\tau)-A(t)>Z^{\varphi}(\tau)+x\right) d x$. From this it can be seen that the novel definitions are related to the weight of the tail, that is the integral of the tail probability which has some practical consequences. The tail probability based approaches try to find a bound on the distribution function of $A(t+\tau)-A(t)$. In our novel approach there is no need for the upper bounds on the entire distribution function, since a bound on the tail weight is enough for the analysis. It will be shown that the expected value form of the definitions provides a simpler interpretation and proof of the statements, due to the linearity of the expected value operator. One

\footnotetext{
${ }^{2}$ Such measures are usually referred to as resource based measures or time-blocking, because they express the probability that a resource (like buffer) is blocked.
} 
the other hand, the expected value approach gives a longrun insight into the behavior of the investigated networks in contrast with the time-instant ${ }^{3}$ view of the saturation probability approaches. Such long term characterization can also be more naturally supported by measurements.

\subsection{Prior Works on Stochastic Network Cal- culus}

The recently developed probabilistic network calculi $[21,2$, $12,8,27$ follow the overflow type description of the inputs and the services similar to inequalities (4) (5) introduced in the previous section. The basic problem of these theoretical studies is the handling of the expressions like the following ${ }^{4}$ : $P\left(\sup _{0 \leq s \leq t}\left\{A(t)-A(t-s)-E^{\varepsilon}(s)\right\}>x\right)$ for arrival processes and $P\left(\sup _{0 \leq s \leq t}\left\{A \otimes S^{\varepsilon}(s)-D(s)\right\}>x\right)$ for services, which require a sample path view.

These expressions can be evaluated by adding some additional assumptions regarding to the arrival and service processes. For example, in [17] it is assumed that a bound is available which limits the length of the busy period over multiple nodes ${ }^{5}$. In [12] the author uses a priori upper bounds , however the derivation of such maximum virtual backlog centric arrival and stochastic service curves that fulfill these bounds is far from trivial. In [27] the authors define a calculus on stochastic processes that have a generalized stochastically bounded burstiness (gSBB) [20], which contains a priori assumptions on the backlog of these processes in a virtual constant rate server. More recently in [8] based on the definition of the stochastically bounded burstiness (SBB [22]), one can observe rate correction terms besides the statistical envelopes and service curves as additional constraints.

\section{A LOSS ORIENTED AND TIME ADAP- TIVE APPROACH}

In this section a new type of arrival and service curve definition are introduced, which relies on bounding the expected deviations from the envelope functions. It will also be proven, that the proposed new descriptors with time adaptivity have the same convenient properties as that of the original ones. The min-plus and max-plus algebra are also applicable to the new functions and the concatenation theorems are also valid. We provide theorems in the new framework to compute such important network descriptors as the backlog or the expected delay. It will be demonstrated how the expectation operator fits naturally into the framework of the network calculus when quantifying important characteristics in long time scales.

If the system is stationary and ergodic the following definition is used for the expected workload loss ratio, $L R$ :

$$
\begin{gathered}
L R=\frac{E[\# \text { of lost bits in a time slot }]}{E[\# \text { of bits arriving in a time slot }]} \leq \\
\frac{E\left[(B-q)^{+}\right]}{E[A]} .
\end{gathered}
$$

${ }^{3}$ The tail probability approach often considers questions like: What is the probability of a buffer overflow at time instant $t$ ?

${ }^{4}$ The difficulties with these expressions are discussed in details in [17].

${ }^{5}$ Such bound is difficult to obtain. where $B$ represents the stationary backlog of the system with infinite buffer, $q$ is the so-called buffer threshold and $E[A]=E[A(0,1)]$ is the number of bits arriving in a unit time slot ${ }^{6}$. We proceed from the definition of the workload loss ratio (6), and based on this, a novel calculus is defined which is suitable for packet loss estimations.

First, the mv-arrival curve ${ }^{7}$, the mv-service curve and their time adaptive versions are defined for describing the inputs and the service, than we prove fundamental per-node statements for the backlog, delay and the mv-arrival curve of the output traffic. Finally, it is shown, that the per-node results can be extended to a network of nodes with the definition of the network mv-service curve. The connection between the mv-arrival curve and effective bandwidth [13], which is a widely used template for resource usage estimation, is also pointed out. For the theorems we assume only the stationarity and ergodicity of the input and output processes.

DeFinition 5 (MV-ARRIVAL CURVE). Let define $Z^{\varphi}$ as the mv-arrival curve of the flow with arrival process $A$ if for all $t, \tau$ and $\varphi \geq 0: E\left[\left(A(t+\tau)-A(t)-Z^{\varphi}(\tau)\right)^{+}\right] \leq \varphi$.

DEFINITION 6 (TIME ADAPTIVE MV-ARRIVAL CURVE). $Z^{\varphi(\tau)}$ is the time adaptive mv-arrival curve of the flow with arrival process $A$ if for all $t$ and $\tau$

$$
E\left[\left(A(t+\tau)-A(t)-Z^{\varphi(\tau)}(\tau)\right)^{+}\right] \leq \varphi(\tau) .
$$

The function $\varphi(\tau)$ is assumed to be positive valued and having a finite sum $\left(\sum_{\tau=0}^{\infty} \varphi(\tau)<\infty\right)$. ${ }^{8}$

We note that the time adaptive mv-arrival curve enables the relaxation of the stronger assumption set about the input processes used in [8]. Now we continue with the definition of the mv-service curve:

Definition 7 (MV-SERVICE CURVE). For an input with arrival process $A$ a node offers an mv-service curve $S^{\varphi_{s}}$ if for all $t \geq 0$ and $\varphi_{s} \geq 0: E\left[\left(A \otimes S^{\varphi_{s}}(t)-D(t)\right)^{+}\right] \leq \varphi_{s}$.

Definition 8 (Time AdAPtive MV-SERvice CuRVe). For an input with arrival process $A$ a node offers a time adaptive $m v$-service curve $S^{\varphi_{s}(t)}$ if for all $t \geq 0$ :

$$
E\left[\left(A \otimes S^{\varphi_{s}(t)}(t)-D(t)\right)^{+}\right] \leq \varphi_{s}(t) .
$$

${ }^{6} \mathrm{It}$ is proven (e.g., in [11] and [14]) that the expected value of the number of lost bits in a finite buffer system can be bounded from above by the number of packets overflown (when the queue size exceeds a certain buffer threshold) in the system with infinite buffer.

${ }^{7}$ The abbreviation mv stands for 'mean value', and is used to clearly differentiate the mv-arrival and mv-service curves from other definitions.

${ }^{8}$ The time dependence of the bounds in [17] and in this paper is fundamentally different. Authors in [17] assumes that there exist an upper bound on the busy period for all sample paths and derives a bound along this assumption (as a consequence the bounds in [17] rely on a busy period analysis). In our paper there is no need to require this property. The construction of the time-adaptive mv-arrival curve achieves that upper bounds can be given for the performance measures without having such assumption. 
Again, $\varphi_{s}(t)$ is assumed to be positive valued and having a finite sum $\left(\sum_{t=0}^{\infty} \varphi_{s}(t)<\infty\right)$.

The ultimate advantage of time adaptive curves is that the non time adaptive versions cannot be used for bounds requiring sample path arguments. In other cases the non time adaptive curves still can be used (e.g., the mv-service curve in single node performance bounds.). Note, that by letting $\varphi$ and $\varphi_{s}$ to zero the arrival and service curves of the deterministic network calculus can be recovered. In other words if $\varphi$ equals to zero, $Z^{\varphi}$ becomes a deterministic arrival curve of the arrival process $A$, and $S^{\varphi_{s}}$ becomes a deterministic service curve if $\varphi_{s}$ equals zero. The previous property involves that the time adaptive curves converges to the deterministic curves as the time goes to infinity since if $\sum_{t=0}^{\infty} \varphi(t)<\infty$ then $\lim _{t \rightarrow \infty} \varphi(t)=0$.

Within the framework of the following theorems we formalize stochastic bounds on some fundamental system characteristics like backlog, delay and output traffic, with minplus calculus operations on mv-arrival curves and mv-service curves. For the proofs the following lemma is needed about the positive part operator:

Lemma 1. For given $X_{1}, X_{2}, X_{3}, X_{4}$ random variables:

$E\left[\left(X_{1}-X_{2}+X_{3}-X_{4}\right)^{+}\right] \leq E\left[\left(X_{1}-X_{2}\right)^{+}\right]+E\left[\left(X_{3}-X_{4}\right)^{+}\right]$

Theorem 1 (BACKLOG BOUND). Let $Z^{\varphi(\tau)}$ be a time adaptive mv-arrival curve of an arrival process $A$ and $S^{\varphi_{s}}$ be a mv-service curve offered by a system that the flow traverses. $Z^{\varphi(\tau)} \oslash S^{\varphi_{s}}(0)$ is a probabilistic bound on the backlog, in the sense that, for all $t \geq 0$,

$$
E\left[\left(B(t)-Z^{\varphi(\tau)} \oslash S^{\varphi_{s}}(0)\right)^{+}\right] \leq \sum_{\tau=0}^{\infty} \varphi(\tau)+\varphi_{s} .
$$

Proof. It follows from the definition of the backlog that $E\left[\left(B(t)-Z^{\varphi(\tau)} \oslash S^{\varphi_{s}}(0)\right)^{+}\right]=E\left[\left(A(t)-D(t)-Z^{\varphi(\tau)} \oslash\right.\right.$ $\left.\left.S^{\varphi_{s}}(0)\right)^{+}\right]=E\left[\left(A(t)+A \otimes S^{\varphi_{s}}(t)-D(t)-A \otimes S^{\varphi_{s}}(t)-\right.\right.$ $\left.\left.Z^{\varphi(\tau)} \oslash S^{\varphi_{s}}(0)\right)^{+}\right]$.

By using Lemma 1 we get:

$E\left[\left(A(t)+A \otimes S^{\varphi_{s}}(t)-D(t)-A \otimes S^{\varphi_{s}}(t)-Z^{\varphi(\tau)} \oslash S^{\varphi_{s}}(0)\right)^{+}\right] \leq$ $E\left[\left(A(t)-A \otimes S^{\varphi_{s}}(t)-Z^{\varphi(\tau)} \oslash S^{\varphi_{s}}(0)\right)^{+}\right]+E\left[\left(A \otimes S^{\varphi_{s}}(t)-\right.\right.$ $\left.D(t))^{+}\right]$.

From the definition of the mv-service curve and by extracting the $A \otimes S^{\varphi_{s}}(t)$ we recover that

$E\left[\left(A(t)-A \otimes S^{\varphi_{s}}(t)-Z^{\varphi(\tau)} \oslash S^{\varphi_{s}}(0)\right)^{+}\right]+E\left[\left(A \otimes S^{\varphi_{s}}(t)-\right.\right.$ $\left.D(t))^{+}\right] \leq E\left[\left(A(t)-\inf _{0 \leq s \leq t}\left\{A(t-s)+S^{\varphi_{s}}(s)\right\}-Z^{\varphi(\tau)} \oslash\right.\right.$ $\left.\left.S^{\varphi_{s}}(0)\right)^{+}\right]+\varphi_{s}$.

It can be easily verified that

$E\left[\left(A(t)-\inf _{0 \leq s \leq t}\left\{A(t-s)+S^{\varphi_{s}}(s)\right\}-Z^{\varphi(\tau)} \oslash S^{\varphi_{s}}(0)\right)^{+}\right]+$ $\varphi_{s}=E\left[\left(\sup _{0 \leq s \leq t}\left\{A(t)-A(t-s)+S^{\varphi_{s}}(s)-Z^{\varphi(\tau)} \oslash S^{\varphi_{s}}(0)\right\}\right)^{+}\right]+$ $\varphi_{s} \leq \sum_{s=0}^{t} E\left[\left(A(t)-A(t-s)-S^{\varphi_{s}}(s)-Z^{\varphi(\tau)} \oslash S^{\varphi_{s}}(0)\right)^{+}\right]+$ $\varphi_{s}$

Now we can increase each term of the sum by the substitution of $s$ into the min-plus deconvolution, so we get:

$\sum_{s=0}^{t} E\left[\left(A(t)-A(t-s)-S^{\varphi_{s}}(s)-Z^{\varphi(\tau)} \oslash S^{\varphi_{s}}(0)\right)^{+}\right]+\varphi_{s} \leq$ $\sum_{s=0}^{t} E\left[\left(A(t)-A(t-s)-S^{\varphi_{s}}(s)-Z^{\varphi(\tau)}(s)+S^{\varphi_{s}}(s)\right)^{+}\right]+\varphi_{s}$. After simplification we get: $\sum_{s=0}^{t} E\left[\left(A(t)-A(t-s)-S^{\varphi_{s}}(s)-Z^{\varphi(\tau)}(s)+S^{\varphi_{s}}(s)\right)^{+}\right]+$ $\varphi_{s}=\sum_{s=0}^{t} E\left[\left(A(t)-A(t-s)-Z^{\varphi(\tau)}(s)\right)^{+}\right]+\varphi_{s}$.

From the definition of the time adaptive mv-arrival curve we conclude that

$\sum_{s=0}^{t} E\left[\left(A(t)-A(t-s)-Z^{\varphi(\tau)}(s)\right)^{+}\right]+\varphi_{s} \leq \sum_{s=0}^{\infty} E[(A(t)-$ $\left.\left.A(t-s)-Z^{\varphi(\tau)}(s)\right)^{+}\right]+\varphi_{s} \leq \sum_{s=0}^{\infty} \varphi(s)+\varphi_{s}$,

which completes the proof.

One may notice that the left hand side of (9) expresses the expected value of the number of bits above a certain buffer level $Z^{\varphi(\tau)} \oslash S^{\varphi_{s}}(0)$ in an infinite buffer system. In other words, in a buffered system with a buffer size $Z^{\varphi(\tau)} \oslash S^{\varphi_{s}}(0)$ the statement in (9) establishes a definition based upper bound on the loss rate. Dividing this loss rate with the expected value of the bits arriving to the node gives a direct upper bound on the workload loss ratio.

TheOREM 2 (MV-ARRIVAl CURVE FOR THE OUTPUT). Let $Z^{\varphi(\tau)}$ be a time adaptive mv-arrival curve of an arrival process $A$ and $S^{\varphi_{s}}$ be a mv-service curve offered by a system that the flow traverses. The function $Z^{\varphi(\tau)} \oslash S^{\varphi_{s}}(t)$ is a mv-arrival curve for the output traffic of the node in the sense that:

$E\left[\left(D(t+u)-D(t)-Z^{\varphi(\tau)} \oslash S^{\varphi_{s}}(u)\right)^{+}\right] \leq \sum_{\tau=0}^{\infty} \varphi(\tau)+\varphi_{s}$.

Proof. $E\left[\left(D(t+u)-D(t)-Z^{\varphi(\tau)} \oslash S^{\varphi_{s}}(u)^{+}\right]=E[(D(t+\right.$ $\left.\left.u)+A \otimes S^{\varphi_{s}}(t)-D(t)-A \otimes S^{\varphi_{s}}(t)-Z^{\varphi(\tau)} \oslash S^{\varphi_{s}}(u)\right)^{+}\right]$. Using Lemma 1 and the fact that $A(t+\tau) \geq D(t+\tau)$ we obtain that:

$E\left[\left(D(t+u)+A \otimes S^{\varphi_{s}}(t)-D(t)-A \otimes S^{\varphi_{s}}(t)-Z^{\varphi(\tau)} \oslash\right.\right.$ $\left.\left.S^{\varphi_{s}}(u)\right)^{+}\right] \leq E\left[\left(A(t+u)-A \otimes S^{\varphi_{s}}(t)-Z^{\varphi(\tau)} \oslash S^{\varphi_{s}}(u)\right)^{+}\right]+$ $E\left[\left(A \otimes S^{\varphi s}(t)-D(t)\right)^{+}\right]$.

From the definition of the mv-service curve and by extracting the $A \otimes S^{\varphi_{s}}(t)$ we obtain:

$E\left[\left(A(t+u)-A \otimes S^{\varphi_{s}}(t)-Z^{\varphi(\tau)} \oslash S^{\varphi_{s}}(u)\right)^{+}\right]+E\left[\left(A \otimes S^{\varphi_{s}}(t)-\right.\right.$ $\left.D(t))^{+}\right] \leq E\left[\left(A(t+u)-\inf _{0 \leq s \leq t}\left\{A(t-s)+S^{\varphi_{s}}(s)\right\}-Z^{\varphi(\tau)} \oslash\right.\right.$ $\left.\left.S^{\varphi_{s}}(u)\right)^{+}\right]+\varphi_{s}$.

It can easily be verified that

$E\left[\left(A(t+u)-\inf _{0 \leq s \leq t}\left\{A(t-s)+S^{\varphi_{s}}(s)\right\}-Z^{\varphi(\tau)} \oslash S^{\varphi_{s}}(u)\right)^{+}\right]+$ $\varphi_{s} \leq E\left[\sup _{0 \leq s \leq t}\left\{A(t+u)-A(t-s)+S^{\varphi_{s}}(s)-Z^{\varphi(\tau)} \oslash\right.\right.$ $\left.\left.\left.S^{\varphi_{s}}(u)\right\}\right)^{+}\right]+\varphi_{s} \leq \sum_{s=0}^{t} E\left[\left(A(t+u)-A(t-s)-S^{\varphi_{s}}(s)-\right.\right.$ $\left.\left.Z^{\varphi(\tau)} \oslash S^{\varphi_{s}}(u)\right)^{+}\right]+\varphi_{s} . \leq \sum_{s=0}^{\infty} E[(A(t+u)-A(t-s)-$ $\left.\left.S^{\varphi_{s}}(s)-Z^{\varphi(\tau)} \oslash S^{\varphi_{s}}(u)\right)^{+}\right]+\varphi_{s}$.

Now each term of the sum is increased by the substitution of $s$ into the min-plus deconvolution:

$\sum_{s=0}^{\infty} E\left[\left(A(t+u)-A(t-s)-S^{\varphi_{s}}(s)-Z^{\varphi(\tau)} \oslash S^{\varphi_{s}}(u)\right)^{+}\right]+$ $\varphi_{s} \leq \sum_{s=0}^{\infty} E\left[\left(A(t+u)-A(t-s)-S^{\varphi_{s}}(s)-Z^{\varphi(\tau)}(u+s)+\right.\right.$ $\left.\left.S^{\varphi_{s}}(s)\right)^{+}\right]+\varphi_{s}$.

After simplification:

$\sum_{s=0}^{\infty} E\left[\left(A(t+u)-A(t-s)-S^{\varphi_{s}}(s)-Z^{\varphi(\tau)}(u+s)+\right.\right.$ $\left.\left.S^{\varphi_{s}}(s)\right)^{+}\right]+\varphi_{s}=\sum_{s=0}^{\infty} E\left[\left(A(t+u)-A(t-s)-Z^{\varphi(\tau)}(u+\right.\right.$ $\left.s))^{+}\right]+\varphi_{s}$.

From the definition of the time adaptive mv-arrival curve it follows that

$\sum_{s=0}^{\infty} E\left[\left(A(t+u)-A(t-s)-Z^{\varphi(\tau)}(u+s)\right)^{+}\right]+\varphi_{s} \leq$
$\sum_{\tau=0}^{s=0} \varphi(\tau)+\varphi_{s} . \quad \square$ 
Theorem 3 (Delay Bound). Let $Z^{\varphi(\tau)}$ be a time adaptive mv-arrival curve of an arrival process $A$ and $S^{\varphi_{s}}$ be a mv-service curve offered by a system that the flow traverses. If $d: Z^{\varphi(\tau)}(u-d) \leq S^{\varphi_{s}}(u)$ (d is considered as a delay threshold) for all $u$ then:

$$
E\left[(A(t-d)-D(t))^{+}\right] \leq \sum_{\tau=0}^{\infty} \varphi(\tau)+\varphi_{s} .
$$

Proof. $E[A(t-d)-D(t)]=E\left[\left(A(t-d)-A \otimes S^{\varphi_{s}}(t)+\right.\right.$ $\left.\left.A \otimes S^{\varphi_{s}}(t)-D(t)\right)^{+}\right]$.

From Lemma 1 it follows that

$E\left[\left(A(t-d)-A \otimes S^{\varphi_{s}}(t)+A \otimes S^{\varphi_{s}}(t)-D(t)\right)^{+}\right] \leq E[(A(t-$ d) $\left.\left.-A \otimes S^{\varphi_{s}}(t)\right)^{+}\right]+E\left[\left(A \otimes S^{\varphi_{s}}(t)-D(t)\right)^{+}\right] \leq E[(A(t-$ d) $\left.\left.-A \otimes S^{\varphi_{s}}(t)\right)^{+}\right]+\varphi_{s}$.

The last step comes from the definition of the mv-service curve. By extracting the min-plus convolution we obtain

$E\left[\left(A(t-d)-A \otimes S^{\varphi_{s}}(t)\right)^{+}\right]+\varphi_{s}=E\left[\left(A(t-d)-\inf _{0 \leq s \leq t}\{A(t-\right.\right.$ $\left.\left.\left.s)+S^{\varphi_{s}}(s)\right\}\right)^{+}\right]+\varphi_{s}$.

Now it can be easily verified that

$E\left[\left(A(t-d)-\inf _{0 \leq s \leq t}\left\{A(t-s)+S^{\varphi_{s}}(s)\right\}\right)^{+}\right]+\varphi_{s} \leq$ $E\left[\left(\sup _{0 \leq s \leq t}\left\{A(t-d)-A(t-s)+S^{\varphi_{s}}(s)\right\}\right)^{+}\right]+\varphi_{s} \leq \sum_{s=0}^{\bar{t}} E[(A(t-$ d) $\left.\left.-A(t-s)+S^{\varphi_{s}}(s)\right)^{+}\right]+\varphi_{s} \leq \sum_{s=0}^{\infty} E[(A(t-d)-A(t-$ $\left.\left.s)+S^{\varphi_{s}}(s)\right)^{+}\right]+\varphi_{s}$.

It follows from the additional assumption of the theorem that

$\sum_{s=0}^{\infty} E\left[\left(A(t-d)-A(t-s)+S^{\varphi_{s}}(s)\right)^{+}\right]+\varphi_{s} \leq \sum_{s=0}^{\infty} E[(A(t-$ $\left.\left.d)-A(t-s)+Z^{\varphi(\tau)}(s-d)\right)^{+}\right]+\varphi_{s} \leq \sum_{\tau=0}^{\infty} \varphi(\tau)+\varphi_{s}$.

The last step follows from the definition of the time adaptive mv-arrival curve.

One can notice that Theorem 3 establishes a bound on the expected value of the number of bits that suffers from a delay larger than $d$. In order to establish end-to-end bounds from the single node results we express the mv-service curve of a network of nodes. In the following theorem the mv-service curve of two concatenated nodes is given.

Theorem 4 (Concatenation of nodes). Assume that a flow traverses nodes $N_{1}$ and $N_{2}$ in sequence. If $E\left[\left(A^{N 1} \otimes\right.\right.$ $\left.\left.S_{N 1}^{\varphi_{1}(t)}(t)-A^{N 2}(t)\right)^{+}\right] \leq \varphi_{1}(t)$ and $E\left[\left(A^{N 2} \otimes S_{N 2}^{\varphi_{2}}(t)-D^{N 2}(t)\right)^{+}\right] \leq$ $\varphi_{2}$, then

$$
E\left[\left(A^{N 1} \otimes S_{N 1}^{\varphi_{1}(t)} \otimes S_{N 2}^{\varphi_{2}}(t)-D^{N 2}(t)\right)^{+}\right] \leq \sum_{t=0}^{\infty} \varphi_{1}(t)+\varphi_{2},
$$

which means, that $S_{N 1}^{\varphi_{1}(t)} \otimes S_{N 2}^{\varphi_{2}}$ is a mv-service curve for the system which consists of the concatenation of these two nodes with $\sum_{t=0}^{\infty} \varphi_{1}(t)+\varphi_{2}$ parameter.

Proof. $E\left[\left(A^{N 1} \otimes S_{N 1}^{\varphi_{1}(t)} \otimes S_{N 2}^{\varphi_{2}}(t)-D^{N 2}(t)\right)^{+}\right]=E\left[\left(A^{N 1} \otimes\right.\right.$ $\left.\left.S_{N 1}^{\varphi_{1}(t)} \otimes S_{N 2}^{\varphi_{2}}(t)-A^{N 2} \otimes S_{N 2}^{\varphi_{2}}(t)+A^{N 2} \otimes S_{N 2}^{\varphi_{2}}(t)-D^{N 2}(t)\right)^{+}\right]$. From Lemma 1 it follows that

$E\left[\left(A^{N 1} \otimes S_{N 1}^{\varphi_{1}(t)} \otimes S_{N 2}^{\varphi_{2}}(t)-A^{N 2} \otimes S_{N 2}^{\varphi_{2}}(t)+A^{N 2} \otimes S_{N 2}^{\varphi_{2}}(t)-\right.\right.$ $\left.\left.D^{N 2}(t)\right)^{+}\right] \leq E\left[\left(A^{N 1} \otimes S_{N 1}^{\varphi_{1}(t)} \otimes S_{N 2}^{\varphi_{2}}(t)-A^{N 2} \otimes S_{N 2}^{\varphi_{2}}(t)\right)^{+}\right]+$ $E\left[\left(A^{N 2} \otimes S_{N 2}^{\varphi_{2}}(t)-D^{N 2}(t)\right)^{+}\right]$.

Using the definition on the min-plus convolution and the mv-service curve we recover that

$E\left[\left(A^{N 1} \otimes S_{N 1}^{\varphi_{1}(t)} \otimes S_{N 2}^{\varphi_{2}}(t)-A^{N 2} \otimes S_{N 2}^{\varphi_{2}}(t)\right)^{+}\right]+E\left[\left(A^{N 2} \otimes\right.\right.$ $\left.\left.S_{N 2}^{\varphi_{2}}(t)-D^{N 2}(t)\right)^{+}\right] \leq E\left[\left(\inf _{0 \leq s \leq t}\left\{\inf _{0 \leq u \leq t-s}\left\{A^{N 1}(t-s-\right.\right.\right.\right.$
$\left.\left.\left.\left.u)+S_{N 1}^{\varphi_{1}(t)}(u)\right\}+S_{N 2}(s)\right\}-\inf _{0 \leq s \leq t}\left\{A^{N 2}(t-s)+S_{N 2}^{\varphi_{2}}(s)\right\}\right)^{+}\right]+$ $\varphi_{2}$.

It can be verified that

$E\left[\inf _{0 \leq s \leq t}\left\{\inf _{0 \leq u \leq t-s}\left\{A^{N 1}(t-s-u)+S_{N 1}^{\varphi_{1}(t)}(u)\right\}+S_{N 2}^{\varphi_{2}}(s)\right\}-\right.$ $\left.\left.\inf _{0 \leq s \leq t}\left\{A^{N 2}(t-s)+S_{N 2}^{\varphi_{2}}(s)\right\}\right)^{+}\right]+\varphi_{2} \leq$

$E\left[\left(\sup _{0 \leq s \leq t}\left\{\inf _{0 \leq u \leq t-s}\left\{A^{N 1}(t-s-u)+S_{N 1}^{\varphi_{1}(t)}(u)\right\}+S_{N 2}^{\varphi_{2}}(s)-\right.\right.\right.$ $\left.\left.\left.A^{N 2}(t-s)+S_{N 2}^{\varphi_{2}}(s)\right\}\right)^{+}\right]+\varphi_{2} \leq \sum_{s=0}^{t} E\left[\left(\inf _{0 \leq u \leq t-s}\left\{A^{N 1}(t-\right.\right.\right.$ $\left.\left.\left.s-u)+S_{N 1}^{\varphi_{1}(t)}(u)\right\}+S_{N 2}^{\varphi_{2}}(s)-A^{N 2}(t-s)-S_{N 2}^{\varphi_{2}}(s)\right)^{+}\right]+\varphi_{2} \leq$ $\sum_{s=0}^{\infty} E\left[\left(\inf _{0 \leq u \leq t-s}\left\{A^{N 1}(t-s-u)+S_{N 1}^{\varphi_{1}(t)}(u)\right\}+S_{N 2}^{\varphi_{2}}(s)-\right.\right.$ $\left.\left.A^{N 2}(t-s)-S_{N 2}^{\varphi}(s)\right)^{+}\right]+\varphi_{2}$.

After simplification we obtain:

$\sum_{s=0}^{\infty} E\left[\inf _{0 \leq u \leq t-s}\left\{A^{N 1}(t-s-u)+S_{N 1}^{\varphi_{1}(t)}(u)\right\}+S_{N 2}^{\varphi_{2}}(s)-\right.$ $\left.\left.A^{N 2}(t-s)-S_{N 2}(s)\right)^{+}\right]+\varphi_{2} \leq \sum_{s=0}^{\infty} E\left[\left(\inf _{0 \leq u \leq t-s}\left\{A^{N 1}(t-\right.\right.\right.$ $\left.\left.\left.s-u)+S_{N 1}^{\varphi_{1}(t)}(u)\right\}-A^{N 2}(t-s)\right)^{+}\right]+\varphi_{2}=\sum_{s=0}^{\infty} E\left[\left(A^{N 1} \otimes\right.\right.$ $\left.\left.S_{N 1}^{\varphi_{1}(t)}(t-s)-A^{N 2}(t-s)\right)^{+}\right]+\varphi_{2} \leq \sum_{t=0}^{\infty} \varphi_{1}(t)+\varphi_{2}$.

The last step follows from the definition of the time adaptive mv-arrival curve.

${ }^{9}$ The application of Theorem 4 iteratively to a network of nodes gives the following corollary.

Corollary 1 (Network MV-Service Curve). If the service offered at each node $h=1, \ldots, H$ on the path of a flow is given by a time adaptive mv-service curve $S_{h}^{\varphi_{\text {sh }}(t)}$ for $h=1, \ldots, H-1$ and by a mv-service curve $S_{H}^{\varphi_{s} H}$ for the $H$-th node, then a network mv-service curve $S_{\text {net }}^{\varphi_{\omega}}$ for the flow is given by

$$
S_{n e t}^{\varphi_{\omega}}(t)=S_{1}^{\varphi_{s 1}(t)} \otimes S_{2}^{\varphi_{s 2}(t)} \otimes \ldots \otimes S_{H}^{\varphi_{s H}}
$$

with a parameter

$$
\varphi_{\omega}=\varphi_{s H}+\sum_{h=1}^{H-1} \sum_{t=0}^{\infty} \varphi_{s h}(t) .
$$

Using Corollary 1 it is possible now to draw up end-to-end workload loss ratio bounds according to Theorem 1 .

\section{THE MV-ARRIVAL CURVE AND THE EF- FECTIVE BANDWIDTH}

The theory of effective bandwidth [13] defines a framework for service provisioning, and as a template (function) it enables computing the bandwidth or buffer requirement for traffic sources in terms of the QoS requirements and the operating point of the buffered system. Hence, this concept provides a measure of resource usage which takes into account the varying statistical characteristics and QoS requirements of traffic sources. The exact definition is recalled here from [13]:

Definition 9 (EFFECTIVE BANDWidth [13]). The effective bandwidth of the source with arrival process $A(t)$ is de-

\footnotetext{
${ }^{9}$ Note that, if we assume the second node also has a timeadaptive curve, a similar (but more difficult to read) proof can be given for the concatenation of the two nodes. This also means that the convolution remains associative in this case.
} 
fined as:

$$
\alpha_{e}(s, \tau)=\sup _{t \geq 0}\left\{\frac{1}{s t} \log E\left[e^{s(A(t+\tau)-A(t))}\right]\right\}, 0<s, \tau<\infty .
$$

The following theorem relates the mv-arrival curve and the effective bandwidth.

THEOREM 5. The mv-arrival curve of an input source can be expressed from it's effective bandwidth, according to the following equation:

$$
Z^{\varphi}(\tau)=\inf _{s>0}\left\{\tau \alpha_{e}(s, \tau)-\frac{\log (\varphi s)}{s}\right\}
$$

Proof. According to exponential bounds on random variables

$$
E\left[\left(A(t+\tau)-A(t)-Z^{\varphi}(\tau)\right)^{+}\right] \leq \frac{e^{s\left(-Z^{\varphi}(\tau)+\tau \alpha_{e}(s, \tau)\right)}}{s}
$$

holds for all positive values of $s$. Let $\varphi$ identified as: $\varphi:=\frac{e^{s\left(-Z^{\varphi}(\tau)+\tau \alpha_{e}(s, \tau)\right)}}{s}$. For $Z^{\varphi}(\tau)$ we obtain:

$$
Z^{\varphi}(\tau)=\tau \alpha_{e}(s, \tau)-\frac{\log (\varphi s)}{s} .
$$

By taking the infimum over $s$ we obtain the smallest mvarrival curve as the right hand side of equation (16).

A similar proof can be given for the following statement about time adaptive mv-arrival curves.

$$
Z^{\varphi(\tau)}(\tau)=\inf _{s>0}\left\{\tau \alpha_{e}(s, \tau)-\frac{\log (\varphi(\tau) s)}{s}\right\}
$$

For demonstration, the time adaptive mv-arrival curve of multiplexed regulated input flows ${ }^{10}$ is shown on Figure 1. The time adaptive mv-arrival curve is normalized by the number of flows and the per flow deterministic arrival curve is also shown for easier interpretation. One can see that the time adaptive mv-arrival curve exploits a significant statistical multiplexing gain.

\section{DETERMINING MV-ARRIVAL AND MV- SERVICE CURVES}

In the followings an illustration is given about the derivation of the mv-arrival and mv-service curves for given inputs and schedulers. For this purpose the mv-arrival curve for regulated arrivals and the mv-service curve of the GPS (Generalized Processor Sharing [19]) scheduler is investigated.

\subsection{MV-arrival curve for Regulated Arrivals}

We may consider regulated inputs as any kind of inputs shaped by a general traffic shaper ${ }^{11}$, e.g., a token bucket controller. This traffic shaper ensures, that the output flow has $\alpha$ as an arrival curve (see Definition 2), where $\alpha$ is a

${ }^{10}$ The mix of collection 1 and 2. See Table 1 in section 7 .

${ }^{11}$ Traffic shaping is frequently used in QoS architectures, e.g., in DiffServ.

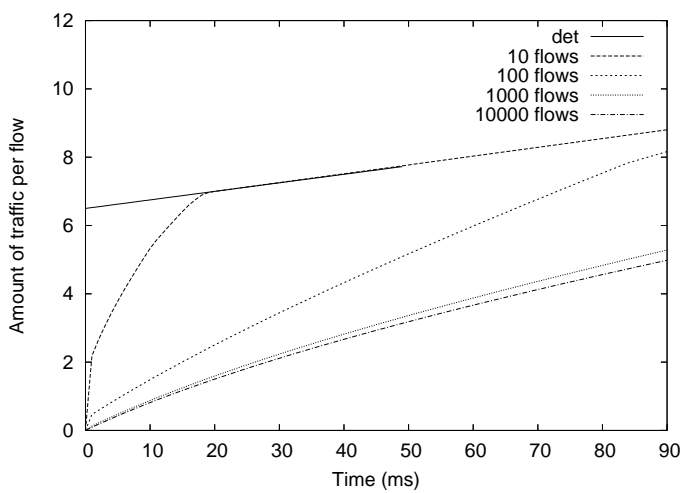

Figure 1: The statistical multiplexing gain.

non-negative wide-sense increasing function. Consider the collection $(\mathcal{I})$ of $I$ inputs and assume, that these inputs are independent and for each $i \in \mathcal{I}$, and for any $s, t \in \mathcal{R}$, $E\left[A_{i}(s, t]\right] \leq \bar{\alpha}_{i} \cdot(t-s)$, where $\bar{\alpha}_{i}=\lim _{t \rightarrow \infty} \alpha_{i}(t) / t<\infty$ is the average rate of the flow. ${ }^{12}$ The effective bandwidth of such an aggregate satisfies [13] the following equation:

$$
\alpha(s, t) \leq \frac{1}{s t} \sum_{i \in \mathcal{I}} \log \left(1+\frac{\bar{\alpha}_{i} t}{\alpha_{i}(t)}\left(e^{\left(s \alpha_{i}(t)\right)}-1\right)\right) .
$$

Therefore in the sense of Theorem 5 the mv-arrival curve is given by

$$
Z^{\varphi}(t)=\inf _{s>0}\left\{\frac{1}{s} \sum_{i \in \mathcal{I}} \log \left(1+\frac{\overline{\alpha_{i}} t}{\alpha_{i}(t)}\left(e^{\left(s \alpha_{i}(t)\right)}-1\right)\right)-\frac{\log (\varphi s)}{s}\right\} .
$$

\subsection{MV-service curve for the GPS Scheduler}

For the proof of the main results in this subsection the following lemma is needed.

Lemma 2. For given $X_{1}, X_{2}, X_{3}, X_{4}$ random variables: $E\left[\left(\left(X_{1}-X_{2}\right)^{+}-\left(X_{3}-X_{4}\right)^{+}\right)^{+}\right] \leq E\left[\left(\left(X_{1}-X_{2}\right)-\left(X_{3}-X_{4}\right)\right)^{+}\right]$

Consider a GPS scheduler with $I$ sessions. Let $Z_{i}^{\varphi}$ be an mvarrival curve of the input from session $i, \psi_{i}$ be the weight of session $i$, and assume that the $Z_{i}^{\varphi}$ functions are concave.

Theorem 6 (GPS scheduler). The curve

$$
S_{i}^{\varphi_{s}}(t)=g_{i} t+\sum_{j \neq i} \frac{\psi_{i}}{\sum_{k=1}^{I} \psi_{k}-\psi_{j}}\left[g_{j} t-Z_{j}^{\varphi}(t)\right]^{+}
$$

is a mv-service curve for the input of session $i$ with $\varphi_{s}=$ $\sum_{j \neq i} \frac{\psi_{i}}{\sum_{k=1}^{I} \psi_{k}-\psi_{j}} \varphi$, where $C$ is the rate of the link represented by the scheduler, and $g_{i}=\frac{\psi_{i} C}{\sum \psi_{j}}$ is the guaranteed

\footnotetext{
${ }^{12}$ The limit $\bar{\alpha}_{i}$ always exists for the wide class of sub additive functions and can be computed as $\sup _{t}\left\{\alpha_{i}(t) / t\right\}$.
} 
minimum service rate for session $i$. Here we note that (23) is the expected value calculus type refinement of the effective service curve derived in [17].

Proof. For simplification in the followings let $\kappa_{j}^{i}=\frac{\psi_{i}}{\sum_{k=1}^{I} \psi_{k}-\psi_{j}}$ The meaning of this coefficient is, that if there is some leftover capacity from session $j$, session $i$ receives the $\kappa_{j}^{i}$-th part of that from the scheduler.

Fix $t \geq 0$ and let $t_{x}=\max \left\{s \leq t: B_{x}(s)=0\right\}$ and $t_{x y}=\max \left\{s \leq t_{x}, x \neq y: B_{y}(s)=0\right\}$. Here $t_{x y}$ can be considered as the latest backlog clearing time point in the system before $t_{x}$. Since:

$B_{x}(t)=A_{x}(t)-D_{x}(t) \leq A_{x}(t)-A_{x}\left(t_{x}\right)-g_{x}\left(t-t_{x}\right)$,

by replacing $t$ with $t_{y}$ and $t_{y}$ with $t_{y x}$ we get:

$D_{x}(t)-D_{x}\left(t_{y}\right) \leq A_{x}(t)-A_{x}\left(t_{y}\right)+B_{x}\left(t_{x}\right) \leq A_{x}(t)-A_{x}\left(t_{y x}\right)-$

$g_{x}\left(t_{y}-t_{y x}\right)$.

From this it follows that:

$D_{y}(t)-D_{y}\left(t_{y}\right) \geq g_{y}\left(t-t_{y}\right)+\sum_{x \neq y} \kappa_{x}^{y}\left[g_{x} t-D_{x}(t)-D_{x}\left(t_{y}\right)\right]^{+} \geq$ $g_{y}\left(t-t_{y}\right)+\sum_{x \neq y} \kappa_{x}^{y}\left[g_{x} t-A_{x}(t)-A_{x}\left(t_{y x}\right)\right]^{+}$,

so finally we obtain that:

$$
\begin{aligned}
& D_{y}(t) \geq A_{y}\left(t_{y}\right)+g_{y}\left(t-t_{y}\right)+ \\
& \quad+\sum_{x \neq y} \kappa_{x}^{y}\left[g_{x}\left(t-t_{y x}\right)-\left(A_{x}(t)-A_{x}\left(t_{y x}\right)\right)\right]^{+} .
\end{aligned}
$$

From the definition of the mv-service curve we obtain $E\left[\left(A_{i} \otimes S_{i}^{\varphi_{s}}(t)-D_{i}(t)\right)^{+}\right] \leq E\left[\left(A_{i}\left(t_{i}\right)+S_{i}^{\varphi_{s}}\left(t-t_{i}\right)-D_{i}(t)\right)^{+}\right]=$ $E\left[\left(A_{i}\left(t_{i}\right)+g_{i}\left(t-t_{i}\right)+\sum_{j \neq i} \kappa_{j}^{i}\left[g_{j}\left(t-t_{i}\right)-Z_{j}^{\varphi}\left(t-t_{i}\right)\right]^{+}{ }_{-}\right.\right.$ $\left.D_{i}(t)\right)^{+}$.

By applying (24) we recover that:

$E\left[\left(A_{i}\left(t_{i}\right)+g_{i}\left(t-t_{i}\right)+\sum_{j \neq i} \kappa_{j}^{i}\left[g_{j}\left(t-t_{i}\right)-Z_{j}^{\varphi}\left(t-t_{i}\right)\right]^{+}-\right.\right.$ $\left.\left.D_{i}(t)\right)^{+}\right] \leq E\left[\left(A_{i}\left(t_{i}\right)+g_{i}\left(t-t_{i}\right)+\sum_{j \neq i} \kappa_{j}^{i}\left[g_{j}\left(t-t_{i}\right)-Z_{j}^{\varphi}(t-\right.\right.\right.$ $\left.\left.t_{i}\right)\right]^{+}-A_{i}\left(t_{i}\right)-g_{i}\left(t-t_{i}\right)+\sum_{j \neq i} \kappa_{j}^{i}\left[g_{j}\left(t-t_{i j}\right)-\left(A_{j}(t)-\right.\right.$ $\left.\left.\left.\left.A_{j}\left(t_{i j}\right)\right)\right]^{+}\right)^{+}\right]$.

After some simplification we get:

$E\left[\left(A_{i}\left(t_{i}\right)+g_{i}\left(t-t_{i}\right)+\sum_{j \neq i} \kappa_{j}^{i}\left[g_{j}\left(t-t_{i}\right)-Z_{j}^{\varphi}\left(t-t_{i}\right)\right]^{+}-\right.\right.$ $\left.\left.A_{i}\left(t_{i}\right)-g_{i}\left(t-t_{i}\right)+\sum_{j \neq i} \kappa_{j}^{i}\left[g_{j}\left(t-t_{i j}\right)-\left(A_{j}(t)-A_{j}\left(t_{i j}\right)\right)\right]^{+}\right)^{+}\right]=$ $E\left[\left(\sum_{j \neq i} \kappa_{j}^{i}\left[g_{j}\left(t-t_{i}\right)-Z_{j}^{\varphi}\left(t-t_{i}\right)\right]^{+}-\left[g_{j}\left(t-t_{i j}\right)-\left(A_{j}(t)-\right.\right.\right.\right.$ $\left.\left.\left.\left.A_{j}\left(t_{i j}\right)\right)\right]^{+}\right)^{+}\right]$.

Since $Z_{i}^{\varphi}$ is concave, the function $\left[g_{j} t-Z_{j}^{\varphi}(t)\right]$ is nondecreasing in $t$. Since $t_{i} \geq t_{i j}$ we can replace $t-t_{i}$ with $t-t_{i j}$ : $E\left[\left(\sum_{j \neq i} \kappa_{j}^{i}\left[g_{j}\left(t-t_{i}\right)-Z_{j}^{\varphi}\left(t-t_{i}\right)\right]^{+}-\left[g_{j}\left(t-t_{i j}\right)-\left(A_{j}(t)-\right.\right.\right.\right.$ $\left.\left.\left.\left.A_{j}\left(t_{i j}\right)\right)\right]^{+}\right)^{+}\right] \leq E\left[\left(\sum_{j \neq i} \kappa_{j}^{i}\left[g_{j}\left(t-t_{i j}\right)-Z_{j}^{\varphi}\left(t-t_{i j}\right)\right]^{+}-\left[g_{j}(t-\right.\right.\right.$ $\left.\left.\left.\left.t_{i j}\right)-\left(A_{j}(t)-A_{j}\left(t_{i j}\right)\right)\right]^{+}\right)^{+}\right]$.

With the usage of Lemma 2 :

$E\left[\left(\sum_{j \neq i} \kappa_{j}^{i}\left[g_{j}\left(t-t_{i j}\right)-Z_{j}^{\varphi}\left(t-t_{i j}\right)\right]^{+}-\left[g_{j}\left(t-t_{i j}\right)-\left(A_{j}(t)-\right.\right.\right.\right.$ $\left.\left.\left.\left.A_{j}\left(t_{i j}\right)\right)\right]^{+}\right)^{+}\right] \leq E\left[\left(\sum_{j \neq i} \kappa_{j}^{i}\left[g_{j}\left(t-t_{i j}\right)-Z_{j}^{\varphi}\left(t-t_{i j}\right)-g_{j}(t-\right.\right.\right.$ $\left.\left.\left.\left.t_{i j}\right)+A_{j}(t)-A_{j}\left(t_{i j}\right)\right]^{+}\right)^{+}\right]=E\left[\sum_{j \neq i} \kappa_{j}^{i}\left[A_{j}(t)-A_{j}\left(t_{i j}\right)-\right.\right.$ $\left.Z_{j}^{\varphi}\left(t-t_{i j}\right]^{+}\right]=\sum_{j \neq i} \kappa_{j}^{i} E\left[\left[A_{j}(t)-A_{j}\left(t_{i j}\right)-Z_{j}^{\varphi}\left(t-t_{i j}\right]^{+}\right] \leq\right.$ $\sum_{j \neq i} \kappa_{j}^{i} \varphi$.

The last step follows from the definition of the mv-arrival curve.

Note, that if we consider a wide-sense decreasing function $\varphi(\tau)$, then a slightly different proof can be given for the following statement about the time adaptive mv-service curve: The curve

$$
S_{i}^{\varphi_{s}(\tau)}(\tau)=g_{i} \tau+\sum_{j \neq i} \frac{\psi_{i}}{\sum_{k=1}^{I} \psi_{k}-\psi_{j}}\left[g_{j} \tau-Z_{j}^{\varphi(\tau)}(\tau)\right]^{+}
$$

is a time adaptive mv-service curve for the input of session $i$ with $\varphi_{s}(\tau)=\sum_{j \neq i} \frac{\psi_{i}}{\sum_{k=1}^{I} \psi_{k}-\psi_{j}} \varphi(\tau)$.

\section{NUMERICAL RESULTS}

In this section we present the results of some numerical investigations in connection with our novel statistical calculus. Let the packets size be fixed to 1500 bytes and let the time be measured in milliseconds. For analysis different flow collections are used. We have four types of input flows which are token bucket constrained with some deterministic arrival curve $\alpha_{i}(t)=\bar{\alpha}_{i} t+\sigma_{i}$. From these input flows we define four flow collections as listed in Table 1 .

\begin{tabular}{|c|c|c|c|}
\hline Coll. & $\bar{\alpha}_{i}$ & $\sigma_{i}$ & Number of flows \\
\hline Coll. 1. & $0.016666 \mathrm{ppms}$ & 5 & 50 \\
\hline Coll. 2. & $0.033333 \mathrm{ppms}$ & 8 & 50 \\
\hline Coll. 3. & $0.066666 \mathrm{ppms}$ & 5 & 50 \\
\hline Coll. 4. & $0.133333 \mathrm{ppms}$ & 8 & 50 \\
\hline
\end{tabular}

Table 1: The summary of collections.

The sustainable rate of the flows are given in packets per milliseconds (ppms) and the maximal burst size is given in packets. These parameter values are close to many practical and common applications, since the flows has 200,400, 800 and $1600 \mathrm{Kbps}$ respectively.

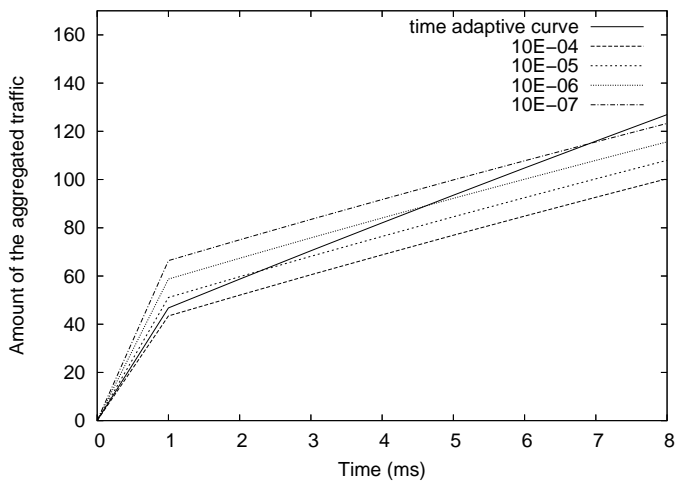

Figure 2: MV-arrival and time adaptive mv-arrival curves.

\subsection{MV-arrival and Time Adaptive mv-arrival curves}

In Figure 2 the comparison of the mv-arrival and the time adaptive mv-arrival curves is shown for the mix of collection 1 and 2. Based on the effective bandwidth for regulated inputs in [13] we used the following formula for the calculation of the time adaptive mv-arrival curves in accordance with Theorem 5:

$$
Z^{\varphi(\tau)}(t)=\inf _{s>0}\left\{\sum_{i \in \mathcal{I}} \frac{1}{s} \log \left(1+\frac{\overline{\alpha_{i}} t}{\alpha_{i}(t)}\left(e^{\left(s \alpha_{i}(t)\right)}-1\right)\right)-\frac{\log (\varphi(\tau) s)}{s}\right\} .
$$

For the mv-arrival curves the setting $\varphi=10^{-4}, \varphi=10^{-5}$ etc. was used, and we set $\varphi(\tau)=e^{-\tau}$ for the time adaptive curve. Figure 2 demonstrates that as the time goes 
to infinity the time adaptive mv-arrival curve suppresses all mv-arrival curves. Despite of this property the time adaptive curves can be efficiently applied in performance bounds referring for example to the workload loss ratio (see subsection 7.2). The success of these newly defined curves relies on the fact that for typical arrival processes the supremum in $Z^{\varphi(\tau)} \oslash S^{\varphi_{s}}(0)$ is attained for small time values that are smaller, than e.g., an upper bound on the busy period.

\subsection{Performance Bounds over Multiple Net- work Nodes}

The scalability of the performance bounds over multiple network nodes is crucial property regarding to the applicability of the novel calculus. Therefore in this section we present loss ratio and delay bounds for the network scenario in Figure 3 .

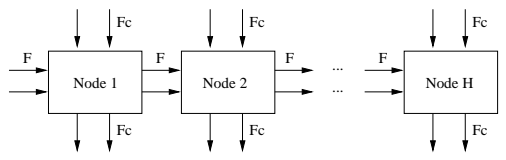

Figure 3: The investigated scenario.

The performance bounds are calculated for the flow collection ( $F$ in Figure 3) that traverses $H$ nodes in sequence. For simplicity at each node the same flow collection will be considered as cross traffic $\left(F_{c}\right)$. Within this analysis a static priority scheduler is used in each node with a setting that the cross traffic $\left(F_{c}\right)$ has higher priority than the flows in $F$. The investigated packet forwarder has a finite capacity and offers service curve $\beta(t)=12.5 \cdot t$, for the aggregate of the input flows in a work-conserving manner. This choice ensures that the packet forwarder has a typical bitrate of $150 \mathrm{Mbps}$.

For the calculation of the time adaptive mv-service curve, which is offered to the aggregate of the flows in $F$ the following statement is used:

Consider a static priority scheduler with $I$ classes. Let $i=$ $1, \ldots, I$ denote the priority of the classes, where the lower number represents higher priority. If we consider a widesense decreasing function $\varphi(\tau)$, then the curve

$$
S_{i}^{\varphi_{s}(\tau)}(\tau)=\left[\beta(\tau)-Z_{j<i}^{\varphi(\tau)}(\tau)\right]^{+}
$$

is a time adaptive mv-service curve for the input of class $i$ with $\varphi_{s}(\tau)=\varphi(\tau)$, where $\beta(\tau)$ is the deterministic service curve offered by the scheduler for the aggregate inputs from all classes, and $Z_{j<i}^{\varphi(\tau)}(\tau)$ is the mv-arrival curve of the aggregate traffic from classes $j<i$. The proof of this statement is similar to the proof of Theorem 6 and it is omitted here.

The calculation of the workload loss ratio happens according to Theorem 1 and the delay according to Theorem 3. For functions $\varphi(\tau)$ and $\varphi_{s}(\tau)$ functions in a form of $K e^{-\theta t}$ have been used and an optimization has been performed according to $K$ and $\theta$.

The logarithm of the $L R$ is shown in Figure 4 as the function of the buffer size, with collection 1 as through flows and collection 2 as cross flows. This mix eventuates a utilization of 0.2 for the scheduler. The curves with different patterns are referring to the different number of network elements.

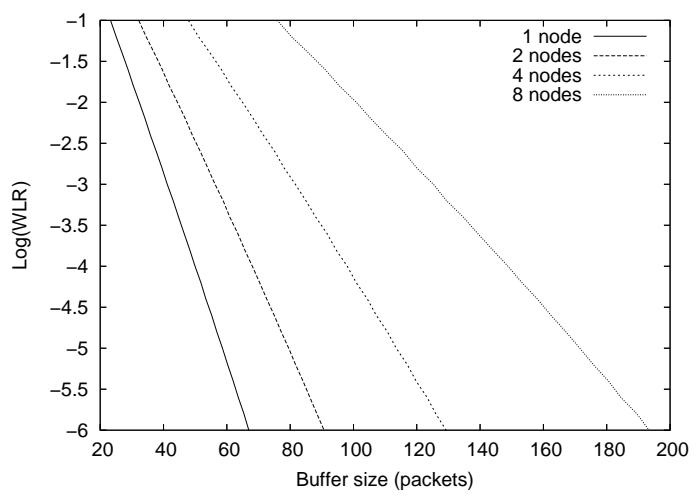

Figure 4: The $L R$ bounds for collection 1 as through flows $(F)$ and collection 2 as cross flows $\left(F_{c}\right)$.

One can see that the buffer requirement of the $L R$ bounds grows rapidly with the increasing number of concatenated network elements. For example for a typical $L R$ value of $10^{-4}$ the buffer requirement is $50,68,98$ and 148 packets for the 1, 2, 4 and 8 node case respectively. From these values the gain of the network service curve becomes plain with respect to the values expected from the node-by-node analysis. The buffer requirement increases significantly when the utilization factor is increased. The same scenario with collection 3 as through and collection 4 as cross flows is shown in Figure 5 for illustration. The mix of collection 3 and 4 produces a utilization of 0.8 for the scheduler. Figure 5 shows how the difference of the buffer requirements for different number of concatenated nodes grows, with the higher utilization. The same phenomenon can be observed by increasing the number of input flows at the schedulers.

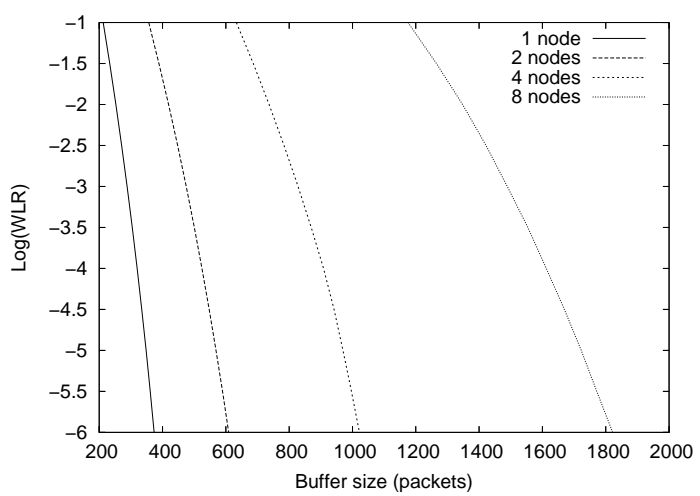

Figure 5: The $L R$ bounds for collection 3 as through flows $(F)$ and collection 4 as cross flows $\left(F_{c}\right)$.

These trends become more obvious when considering delay bounds. For illustration, the logarithm of the expected number of packets that suffer a delay larger than a specific delay value is plotted in Figure 6 From Figure 6 one can see, that 


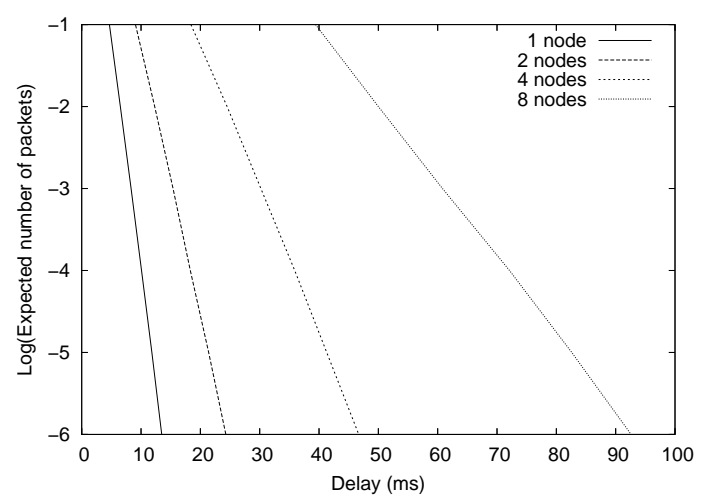

Figure 6: The delay bounds for collection 1 as through flows $(F)$ and collection 2 as cross flows $\left(F_{c}\right)$.

for example $10^{-2}$ packets per milliseconds will suffer a delay of $6.5,12.3,24.5$ and 50 milliseconds in the $1,2,4$, and 8 node case respectively.

\subsection{MV-service curves for the GPS Scheduler}

Finally we should like to illustrate that in case of some schedulers, tighter stochastic service curves (or service envelopes) can be derived by taking into account the special properties of the scheduling algorithm. Theorem 6 presents a mvservice curve for the GPS scheduler in a concise closed form. The mv-service curve there consists of the guaranteed minimum service rate of a given input flow and a term which a lower estimates the capacity left by the sessions that already cleared their backlog. However, as it is shown in [19], the scheduling gain can be much larger than its estimate based on Theorem 6. In some cases the difference can be as large as that the deterministic service curve suppresses the stochastic curve. Figure 7 and 8 illustrate this kind of tradeoff between the scheduling and multiplexing gain.

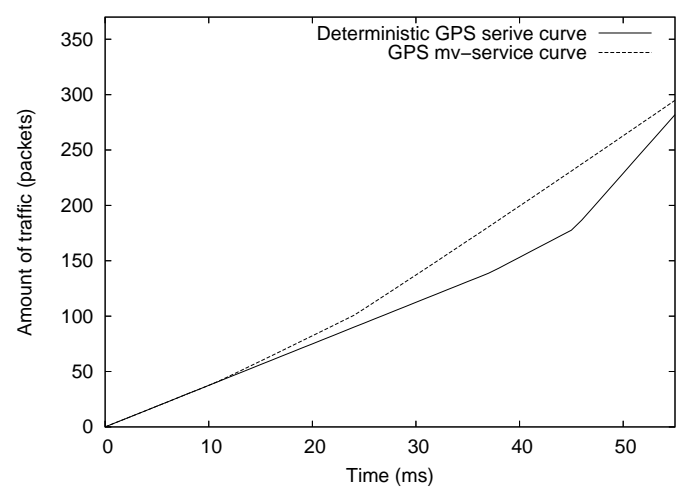

Figure 7: The mv-service and the deterministic service curve offered by the GPS scheduler for a specific session in a scenario with significant multiplexing gain.

Figure 7 shows the mv-service and the deterministic service curve offered by the GPS scheduler for a specific session in a scenario where each session contains a large number of (micro) flows to produce a significant multiplexing gain. One can see that despite of the loose estimation of the scheduling gain the resulting mv-service curve is always larger than the deterministic curve for a typical setting of $\varphi_{s}=10^{-5}$. Figure 8 shows the same curves of a modified scenario, where in one session we replaced the flows with their deterministic aggregate to ignore the statistical multiplexing gain. In this case the deterministic curve becomes larger than the stochastic one over a specific time interval.

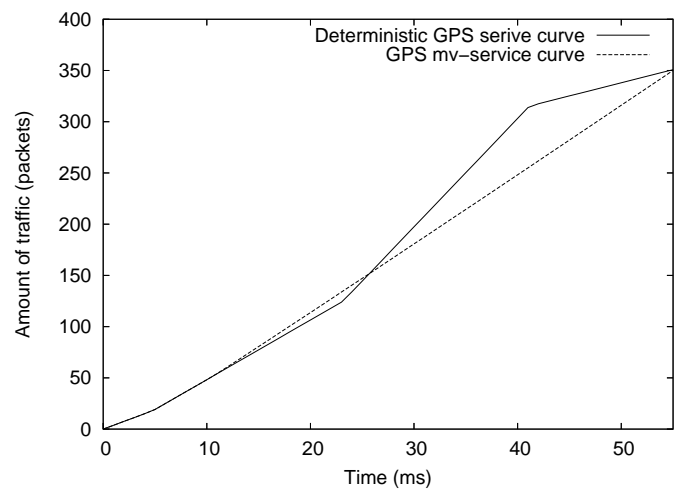

Figure 8: The mv-service and the deterministic service curve offered by the GPS scheduler for a specific session in a scenario with negligible multiplexing gain.

The incorporation of the scheduling gain to the stochastic service curves can be one of the future works on the stochastic service curves.

\section{CONCLUSIONS}

In this paper a novel stochastic network calculus is defined which inherently relates to loss performance analysis thanks to the mean value like definitions of arrival and service curve. Time adaptive versions of this calculus can resolve sample path problems and avoid the use of additional restrictive assumptions used in prior works. Fundamental results have been proven for the backlog, delay and output traffic envelope. The power of this approach is also demonstrated through analytical results and numerical examples for generalized processor sharing (GPS) schedulers.

\section{REFERENCES}

[1] D. D. Botvich and N. G. Duffield. Large deviations, the shape of the loss curve, and economies of scale in large multiplexers. 20:293-320, 1995.

[2] A. Burchard, J. Liebeherr, and S. D. Patek. A calculus for end-to-end statistical service guarantees. Technical Report CS-2001-19, University of Virginia, May 2002.

[3] C. S. Chang. Stability, queue length and delay of deterministic and stochastic queueing networks. IEEE Transactions on Automatic Control, Vol.39, pages: 913-931, 1994.

[4] C. S. Chang. On deterministic traffic regulation and service guarantees: A systematic approach by filtering. 
IEEE Transactions on Information Theory, Vol. 44, pages: 1097-1110, May 1998.

[5] C. Courcoubetis, V. A. Siris, and G. D. Stamoulis. Application of the many sources asymptotic and effective bandwidths to traffic engineering. 12:167-191, 1999.

[6] C. Courcoubetis and R. Weber. Buffer overflow asymptotics for a buffer handling many traffic sources. 33:886-903, 1996.

[7] N. G. Duffield, J. T. Lewis, N. O'Connel, R. Russel, and F. Foomey. Entropy of atm traffic streams: tool for estimating qos parameters. IEEE Journal of Selected Areas in Communications 13(6): 981-990, 1995.

[8] A. B. F. Ciucu and J. Liebeherr. Scaling properties of statistical end-to-end bounds in the network calculus. IEEE Transactions on Information Theory 52(6): 2300 - 2312, June 2006.

[9] M. Fidler. An end-to-end probabilistic network calculus with moment generating functions for efficient utilization of independence. Proceedings of NTNU, Trondheim, Norway, 2005.

[10] A. Gulyás and J. Bíró. A stochastic extension of network calculus for workload loss examinations. IEEE Communications Letters, Volume 10, p. 399-402., 2006.

[11] A. György and T. Borsos. Estimates on the packet loss ratio via queue tail probabilities. In proceedings of IEEE Globecom Vol. 4 pages: 2407-2411, March 2001.

[12] Y. Jiang. A basic stochastic network calculus. ACM SIGCOMM Computer Communication Review 36(4): 123 - 134, October 2006.

[13] F. P. Kelly. Notes on effective bandwidth. Stochastic Networks: Theory and Applications pages: 141-168 Oxford University Press, 1995.

[14] H. Kim and N. B. Shroff. Loss probability calculations and asymptotic analysis for finite buffer multiplexers. IEEE/ACM Transactions on Networking, 9(6): 755 768, Dec 2001

[15] M. Krunz and A. M. Ramasamy. The correlation structure for a class of scene-based video models and its impact on the dimensioning of video buffers. IEEE Transactions on Multimedia 2(1): 27-36, 2000.

[16] J.-Y. Le Boudec and P. Thiran. Network Calculus: A theory of deterministic queuing systems for the Internet. Springer, 2002.

[17] C. Li, A. Burchard, and J. Liebeherr. A network calculus with effective bandwidth. Technical Report CS-2003-20, University of Virginia, November 2003.

[18] M. Montgomery and G. de Veciana. On the relevance of time scales in performance oriented traffic characterizations. volume 2, pages 513-520, San Francisco, USA, March 1996.

[19] A. K. Parekh and R. G. Gallagher. A generalized processor sharing approach to flow control in integrated services networks. IEEE/ACM Transactions on Networking, 1(3):344-357, June 1993.

[20] S. J. Q. Yin, Yuming Jiang and P. Kong. Analysis on generalized stochastically bounded bursty traffic for communication networks. In Proceedings of IEEE Local Computer Networks pp. 141-149, November
2002.

[21] A. B. R. R. Boorstyn, J. Liebeherr, and C. Oottamakorn. Statistical service assurances for traffic scheduling algorithms. IEEE Journal on Selected Areas in Communications 18(12): 2651-2664, December 2000.

[22] D. Starobinski and M. Sidi. Stochastically bounded burstiness for communication networks. IEEE Transactions on Information Theory, Vol. 46, No. 1, pages: 206-212, 2000.

[23] M. Vojnovic and J. Y. Le Boudec. Stochastic analysis of some expedited forwarding networks. Technical Report DSC/2001/039, EPFL-DI-ICA, July 2001.

[24] M. Vojnovic and J.-Y. Le Boudec. Stochastic analysis of some expedited forwarding networks. In proceedings of INFOCOM Vol. 2 pages: 1004-1013, June 2002.

[25] M. Vojnovic and J.-Y. Le Boudec. Bounds for independent regulated inputs multiplexed in a service curve network element. IEEE Transactions on Communications 51(5): 735-740, May 2003.

[26] C. Walsh and N. G. Duffield. Predicting QoS parameters for ATM traffic using shape-function estimation. In Fourteenth UK Teletraffic Symposium, Manchester, UK, March 1997.

[27] C. T. Y. Liu and Y. Jiang. A stochastic network calculus. Technical Report ECE-CCN-0301, Dept. of Electrical and Computer Engineering, National University of Singapore, November 2003. 